\title{
Wikipedians' Knowledge and Moral Duties
}

\author{
Jens-Erik Mai
}

\section{Bio}

Jens-Erik Mai is professor of information studies at the University of Copenhagen. His work concerns basic questions about the nature of information phenomena in contemporary society - he is concerned with the state of privacy and surveillance given new digital media, with classification given the pluralistic nature of meaning and society, and with information and its quality given its pragmatic nature. He teaches courses on classification, design of controlled vocabularies, information ethics, privacy and surveillance, knowledge media, and the theoretical foundation of information studies. He earned his Ph.D. in Library and Information Science from the The School of Information at the University of Texas at Austin as a Fulbright Scholar and his Master and Bachelor degrees from the Royal School of Library and Information Science, Denmark.

\begin{abstract}
Artiklen analyserer erkendelsesteoretiske og etiske forpligtelser $i$ Wikipedias Neutral Point of View (NPOV) politik. Analyserne afslører revner i NPOV politikkens konceptuelle fundament i forhold til begreber som mening, fakta, viden, sikkerhed, tvivl,
\end{abstract}

Jens-Erik Mai, Professor

University of Copenhagen, Information Studies jemai@hum.ku.dk og kognitiv autoritet. Ydermere diskuterer artiklen NPOV politikkens etiske position som antagende en absolutistisk kerne og etisk relativisme ved kanterne. Artiklen konkluderer, at Wikipedia burde genkonceptualisere og omskrive NPOV politikken og erkende, at videns lokalitet er vasentligt, at de sprogspil Wikipedianere er en del af er vosentlige, at viden er som en rhizom med inkommensurable punkter, at udfordringerne ved inklusion ligger $i$ kernen og ikke ved kanterne, og eksplicit tage en etisk pluralistisk position $i$ sit foretagende.

\section{Introduction}

There are currently about five million entries in the English language version of Wikipedia. It is unknown how many of these entries violate Wikipedia's fundamental principle that "All encyclopedic content on Wikipedia must be written from a neutral point of view", which Wikipedia takes to mean "representing fairly, proportionately, and, as far as possible, without bias, all of the significant views that have been published by reliable sources on a topic" (Wikipedia, n.d.-a). However, to address this question of whether specific entries in Wikipedia adhere to Wikipedia's policy and guidelines, there are a number of more foundational questions that first need to be explored. These foundational questions include whether it is possible - or even desirable - to write an encyclopedia article from a neutral point of view, whether a listing of all "significant" views on a topic amounts to neutrality, and, whether knowledge is always po- 
litically, ethically, and culturally bound by context and use.

In this paper I will analyze the epistemological and ethical foundation of Wikipedia's Neutral Point of View (NPOV) policy (Wikipedia, n.d.-a) and argue that it needs to be reconceptualized. I will suggest that Wikipedia ought to acknowledge that homogeneity among Wikipedians poses a challenge to the continuing status of Wikipedia as a cognitive authority. And secondly, I argue that Wikipedia ought to seriously consider that the locality of knowledge and the plurality of understandings, norms' and values should drive Wikipedians' productions of encyclopedic information and Wikipedia entries.

\section{Wikipedia's NPOV policy}

Wikipedia's 4500 words-long policy on Neutral Point of View (NPOV) suggests that the basic principle of NPOV is not to describe a given topic neutrally and objectively. The basic principle is that Wikipedia entries on a given topic should be a reflection of the multiple sides from which the topic can be viewed and how it has been discussed in multiple, reliable sources. The central idea is that Wikipedia should not take sides in debates about the issues, but merely describe the various sides on an issue. The neutrality of Wikipedia rests on the idea it is not possible to describe a given topic objectively, but that it is possible to describe the various sides without bias. Wikipedia states that "to be neutral is to describe debates rather than engage in them", and it advises that entries in Wikipedia are not neutral in the sense of a "'view from nowhere' (to use Thomas Nagel's phrase)" (Wikipedia, n.d.-b).

In The View from Nowhere, Thomas Nagel (1986) discusses the philosophical interrelations and differences between a subjective standpoint and an objective standpoint. He regards a subjective standpoint as one that relies on "the specifics of an individual's makeup and position in the world" (p. 5); as such, a subjective standpoint is one's private view on the world, so to speak. An objective standpoint is one that is "created by leaving a more subjective, individual, or even just human perspective behind" (p. 7); it is a view of the world that is "centerless" (p. 60) and understands the world "from no particular point of view" (p. 61). However, this discussion between subjective and objective standpoints is not the discussion that the NPOV policy engages in. The purpose of the NPOV policy is precisely not to foster a "view from nowhere", but merely to discourage Wikipedians from promoting subjective standpoints. Again, the basic point is that Wikipedia does not aim towards or believe in a neutral, objective "view from nowhere" but aims to be neutral and unbiased with regards to the descriptions of the various viewpoints on the subject matter for entries in Wikipedia.

To enable Wikipedians to describe the various sides on a topic, the NPOV policy distinguishes between "significant viewpoints", "the majority viewpoint", "a small minority" viewpoint, and "a tiny minority" viewpoint (Wikipedia, n.d.-a). Wikipedians shall describe a topic according to the various views on the topic as they have been discussed and described in reliable sources, and Wikipedians shall give the various views their relative weight in the entry. This shall be done such that "all significant viewpoints" (Wikipedia, n.d.-a) are represented with the most weight given to the majority viewpoint and such that the reader understands how small minority viewpoints differ from the majority viewpoint. However, the "views of tiny minorities should not be included at all" (Wikipedia, n.d.-a). To identify these views and determine their relative prominence, Wikipedians are referred to "published, reliable sources", which can be found in libraries where one can consult "reputable books and journal articles." One can consult "reliable online resources" as well (Wikipedia, 2015a). To obtain help identifying "high-quality sources", one can ask the Wikipedia editors or "at the reference desk" in a library (Wikipedia, n.d.-a). ${ }^{1}$ The foundational premise in the NPOV policy is that a topic can be understood and described from various viewpoints - subjective as well as objective - and that there is a majority viewpoint on the topic that is supplemented by small and tiny minority viewpoints. Wikipedians should detach themselves from the subject matter of the topic by relying on information resources that describe facts about the topic, the majority viewpoint, and the various small minority views.

The NPOV policy makes a sharp distinction between opinions and facts. When Wikipedians consult resources on a topic, the NPOV policy advises them to attribute "opinions" (Wikipedia, n.d.-a) to the particular resources in which the opinions are expressed, whereas "uncontested and uncontroversial factual assertions" (Wikipedia, n.d.-a) can be stated 
in "Wikipedia's voice" (Wikipedia, 2015a), as if it were a fact that Wikipedia had verified. If Wikipedians find "conflicting assertions" in multiple reliable resources, these should be treated as "opinions rather than facts" (Wikipedia, n.d.-a). In other words, in Wikipedia an assertion can either be an opinion or a fact. However, only uncontested and uncontroversial assertions can be facts. If there is a discussion or disagreement about a matter in reliable sources, then all assertions are opinions. There cannot be two conflicting facts about a matter.

The neutrality of Wikipedians is achieved by remaining detached and neutral vis-a-vis the topics, by relying on descriptions of debates about the topics in reliable resources, and by focusing on facts. The individual Wikipedians' subjective "makeup and position in the world" (Nagel, 1986, p. 5) therefore matters not, because the aim is to "describe disputes, but not engage in them" (Wikipedia, n.d.-a). Given that topic entries are made up of facts and descriptions of the majority viewpoint and the various small minority views on the topic, each entry in Wikipedia should remain independent of Wikipedians' gender, sexuality, and ethnicity, or their political views, nationality, and religion. In other words, if Wikipedia were to acknowledge or recognize that homogeneity among Wikipedians challenges the kind of knowledge that Wikipedians produce and capture in Wikipedia, Wikipedia first needs to acknowledge that Wikipedians cannot escape their individual makeup and positions in the world. The current version of the NPOV policy does not acknowledge that homogeneity among Wikipedians challenges the kind of knowledge that Wikipedia produces. I will in the following discuss this foundational assumption that Wikipedia's NPOV policy rests upon.

\section{Knowing knowledge}

As it stands, the NPOV policy advances a conceptual position that sits somewhere between Nagel's (1986) subjective standpoint and objective standpoint. Wikipedia aims at being neutral in the sense that it does not engage in the topics at a personal, subjective level nor does it aim to describe topics objectively from a "view from nowhere" position. Instead, the NPOV policy assumes Wikipedians take a position of being an "authority on authorities" (Wilson, 1986, p. 179), one who can determine which resources can be trusted on a given topic, which resources are of high- quality, which views are in minority, which views can be ignored, and which view is the majority view. As such a Wikipedian is one,

"who could be trusted to tell us who else can be trusted, in all possible spheres; such a person would be potentially an authority on everything, for if he could identify the authorities in any sphere, he could in principle find out what they claim to know and so inform himself on any subject what-ever, and subsequently inform us. $\mathrm{He}$ could find out literally anything" (p. 179-180).

In his classic work on the presumed authority of libraries and librarians, Patrick Wilson (1983) famously noted that beyond the narrow scope of our own personal experiences, what "we think about the world is what we have second hand from others" (p. 10) - "it is all hearsay" (p. 13) - but not all hearsay is "equally reliable", because while "some people know what they are talking about, others do not. Those who do are my cognitive authorities" (p. 13). They are cognitive authorities because we let them influence our thinking on specific topics. Like libraries and librarians, Wikipedia and Wikipedians ought to strive to become cognitive authorities, that is institutions and people we turn to for advice and information, and whom we let influence our thinking.

Wilson operates with two kinds of authority: administrative authority and cognitive authority. Administrative authority is limited to the "recognized right to command others, within certain prescribed limits" but importantly this sort of authority does not include the authority "to tell people what to think" (p. 14). Cognitive authority, on the other hand, is of more interest to the discussion of knowledge production and knowledge distribution. Cognitive authorities are those who have "influence on one's thoughts that one would consciously recognize as proper" (p. 15). ${ }^{2}$ In other words, cognitive authority is the special kind of authority that we as receivers of knowledge and information ascribe to the resources of information and knowledge; we can choose to trust the resources. In sum, cognitive authority can be described by three basic characteristics: 1) cognitive authority always exist in a relation involving at least two people. No one can be a cognitive authority all by himself; someone has to recognize the person as a cognitive authority. 2) Some have a lot of cognitive authority, others less so. 3) Cognitive authority is relative 
to specific spheres; one may speak with cognitive authority on some matters, but not necessarily on others.

While the NPOV policy distinguishes between fact and opinion, Wilson distinguishes between knowledge and opinion, which he takes to be the distinction between open and closed questions. Closed questions are those that, for all practical purposes, may be considered as settled: those which we do not ordinarily question or seriously doubt. Sometimes a question that was previously closed may be opened up again, and once again debated in the literature and public discourse. Open questions are those about which there are competing answers, competing avenues for addressing the problem, and differences in strategies in determining proper answers.

Determining whether a particular question is closed or open may "itself be a closed question, but it may also be wide open" (Wilson, 1983, p. 17) and sometimes we may turn to our cognitive authorities to determine exactly this - whether a question is open or closed, and what the arguments are pro and con regarding the question as closed. Wittgenstein (1969) was likewise concerned with this distinction; he asked: "Is it not difficult to distinguish between the cases in which I cannot and those in which I can hardly be mistaken? Is it always clear to which kind a case belongs?" Wittgenstein's answer is: "I believe not" (p. 89e).

Herein lies the foundational challenges with the NPOV policy: where Wikipedia suggests that assertions about the world can be divided into those that are uncontested and universally correct and those that are merely opinions, both Wilson and Wittgenstein believe that the distinction is not as clear. Wittgenstein explores the foundation upon which we can claim to know things, which, like Wilson, he also distinguishes between things we can know first-hand ("here is one hand" (Wittgenstein, 1969,p. 2e)) and things we know second-hand ("the earth is round" (p. 38e)) and in both kind of situations we can "act with complete certainty" (p. 25e). The distinction between the two kinds of knowing might therefore be less interesting and significant. For Wittgenstein the notion of knowledge and to know something is coupled with language games in which the knowledge is used and employed. To know something is to be part of a language game and "it must be something that, by general consent, he is in a position to know" (p. 73e). The notion of knowledge is therefore tightly connected to the notion of certainty; there can only be knowledge where there is also uncertainty. If there is no uncertainty about something, then we cannot really know that thing. And it would not be material for an encyclopedia. The knowledge that is included in an encyclopedia is the kind of knowledge about which one can claim: "I know that...", and one can only claim to 'know that' where there exists some degree of uncertainty, and where mistakes are possible. In other words, the sharp distinction between facts or knowledge on one hand and opinions on the other hand is problematic because, "what I know, I believe" (p. 25e). This does not mean that we cannot be certain of anything, quite the opposite - because the "game of doubting itself presupposes certainty" (p. $18 \mathrm{e}$ ). We are part of language games in which facts are established with certainty that cannot be doubted, and we build our doubt upon those facts - until we start questioning those facts and believing in others, of course.

This establishment of doubts, certainty, and knowledge takes place through language and in the production of texts. As Umberto Eco (1984) states, "a natural language is a flexible system of signification conceived for producing texts, and texts are devices for blowing up or narcotizing pieces of encyclopedic information" (p. 80). Eco suggests that the best way to think about an encyclopedia and the competencies that are required to navigate, read, and understand an encyclopedia is to consider the knowledge network as a rhizome. In the rhizome every point can be connected with every other point; there is no top, no bottom, no beginning, no end. Everything can potentially connect with everything else. It has "unlimited territory" (p. 81). It is not possible to provide a global description or even conceive of the whole rhizome because of its multidimensionality and constant changes. Such a conceptualization of an encyclopedia assumes a network of interpretants to be infinite and to include "multiple interpretations realized by different cultures" (p. 83). Such an encyclopedia would not only register true facts about a topic, but also register what is believed to be false and how different cultures conceive of the topic.

The challenge for the encyclopedia is on one hand to accept the existence of structured knowledge, while at the same time provide only "'local' and transitory 
systems of knowledge, which can be contradicted by alternative and equally 'local' cultural organizations; every attempt to recognize these local organizations as unique and 'global' - ignoring the impartiality - produces an ideological bias" (Eco, 1984, p. 84). Recognizing the locality of knowledge creates the realization that knowledge cannot be reduced to the descriptions of facts, and the listing of series of minority viewpoints without producing an ideological cultural colonialism.

\section{Moral duties}

The NPOV policy's moral foundation is found in its insistence that the aim is to "describe disputes, but not engage in them" (Wikipedia, n.d.-a). However, if the argument in the previous section of this paper is accepted (that Wikipedians are already part of language games and cultures that form the disputes, and as such cannot claim independence from these) then we need to consider Wikipedians' obligations to represent knowledge in a fair manner.

We could read the NPOV policy's stipulation to describe the majority viewpoint and the minority viewpoints as taking a pluralistic view of knowledge. It could be argued that by aiming to represent all the significant viewpoints on a topic that Wikipedia takes the position that it acknowledges that there is no true, objective view of the topic and that there can exist several incommensurable views on the topic. However, if this is indeed a pluralistic standpoint, it is a standpoint that "admits pluralism to be true at the center" and one that "claims that [plurality] only needs to be dealt with at the edges" (Smith, 1996, p. 109). This notion of plurality is present in the NPOV policy's determination that there is a majority viewpoint on a given topic - which can be presented in Wikipedia's voice - and that less space shall be allocated to descriptions of small minority viewpoints. It is also present in the idea that tiny minority viewpoints can be ignored completely. The basic idea is that the center, the majority viewpoint, is true. The NPOV policy's take on plurality is therefore something like:

"yes, sure enough, the words or concepts of a given people or society are not stable or God-given, but [it] nonetheless maintains that they are relatively stable across some identifiable (and expensive) unit of analysis: stable across a populace, say, or a culture, or across a given historical period" ( $\mathrm{p}$. 109).

The danger of this sort of pluralism - which Smith calls "pluralism-in-the-large" (Smith, 1996, p. 109) - is that is fosters an 'I like ice cream', 'I don't' sort of ethics, where people can "claim allegiance to a degree of cultural sensitivity" (p. 112) but it does not really matter. It allows people to acknowledge cultural sensitivity and differences while at the same time to ignore these differences as something that is "extra-theoretical" (p. 112) - as if the "cultural and situational dependence were an independent parameter, to be set once for a given language or people or period of history, and thereafter ignored" (p. 112). This sort of pluralism lets us merely acknowledge that there are differences between cultures and understandings of the world, but it fosters no conversation about these differences; we can learn nothing from the differences because this sort of pluralism does not make the diversity a focus for its ethics. The ethical locus is at the center of truth, and the plurality is limited to the edges.

Another kind of plurality is "dealt with at the center" (Smith, 1996, p. 109) where the discussions and understandings of a topic are:

"constantly lived and multiply textured: a dynamic, day-by-day, in-the-rough, wrestling and struggling with the fit of one's concepts and actions and thoughts into the world surrounding them - fit with the rest of one's belief and hopes and desires, fit with the ideas and desires of one's fellows, fit with with one's community and history - and by no means least, fit with the subject matter they are about" (p. 108)

This sort of pluralism - Smith calls it pluralism-inthe-small (p. 109) - does not subscribe to the idea of a majority view and several minority views. Instead it acknowledges that that meanings and understandings are not stable across whole cultures, but are "a kind of negotiated plasticity" (p. 108). Their most important quality is that of locality: "justice to the particular, the specific, the located" (p. 109).

While this sort of approach to realism and ethics runs the danger of ending up with an "anything goes" kind of relativism, the point here is, in Smith's (1996) terms, "to view knowledge as inexorably situated, 
located, embodied, perspectival, contested, and so forth" (p. 107) and as such hold people accountable to their claims. This approach gives up on objectivity as a view from nowhere, where the world is viewed as something that exists independently of the experiencing subject. On the other hand, it does not take Nagel's subjective position as knowledge aligning "the specifics of an individual's makeup and position in the world" (Nagel, 1986, p. 5). It is a position of realism where "intentional subjects, including us, are full-blooded, embodied, effective participants in the world" (Smith, 1996, p. 97-98) and where we participate in the world through language games. As such there is a break down of the distinction between object and subject, which is assumed in traditional objectivism (as in the view from nowhere position), in Nagel's notion of the subjective standpoint as one's private view on the world, and in the NPOV policy's notion of describing disputes without engaging in them. In these positions there is a distinction between object and subject; the subject experiences and gains knowledge about the object. However, the position advocated here is that both object and subject belong to the same world, and as such they cannot be viewed as independent.

While the NPOV policy explicitly rejects a traditional view from nowhere position, it assumes an equally politically dangerous ethical position. The NPOV policy assumes that the center - the topic for the Wikipedia entry - exists as a value-neutral space in which there can be a majority viewpoint, and that a number of minority cultures have alternative, outlying viewpoints on the topic. It is an ethical position that is absolutistic at the center, but relative at the edges. Wikipedia assumes that the center exists in a space that is "uncontested and uncontroversial" (Wikipedia, n.d.-a), whereas there is only room for "conflicting" "opinions" (Wikipedia, n.d.-a) at the edges.

Both absolutism and relativism are established ethical traditions - and both are unattractive. Ethical relativism "tolerate(s) any and all practices" (Ess, 2009 , p. 193) and as such hinders a stance on a given ethical dilemma, since the practice can be attributed to particular cultural norms and habits and as such is beyond discussion in any other culture than its own. Ethical absolutism, on the other hand, results "in a kind of ethical colonialism" (p. 194) because it imposes a specific set of ethical norms on all people regardless of culture, norms, beliefs, and habits. The NPOV policy incorporates both ethical positions in its framework: one at the center and the other at the edges. The policy assumes that the majority viewpoint is value-neutral (as does all absolutistic ethics) while it can do nothing else but merely present minority views because they are culturally bound and therefore beyond comprehension within the majority view. The NPOV policy thereby takes an ethical absolutistic position on the majority viewpoint at the center of the Wikipedia entry, which is represented in Wikipedia's own voice and presented as being factual and true. Minority views, on the other hand, are dealt with at the edges, regarded as being outside of mainstream culture and outside the majority viewpoint. As such, the minority views are presented as opinions that are tolerated but impossible to fit into the majority, mainstream cultural views.

As an alternative approach, ethical pluralism offers to deal with the representation of knowledge in Wikipedia in a manner that the current NPOV policy strives for, but does not accomplish. The basic idea of ethical pluralism is to take a third approach, alternative to the two opposing positions of ethical absolutism and ethical relativism. Ethical pluralism accepts the first principle of ethical absolutism: that there are in fact basic universal norms, values, and practices across all cultures-yet at the same time it departs from absolutism's second principle: that the same norms, values, and practices can be applied to all cultures. And, while ethical pluralism does agree with ethical relativism on the empirical fact that there are indeed difference practices in different times and cultures, it assumes that these differences are the result of different interpretations of the shared values and norms. As such, the ethical pluralist would find that "it is possible (indeed, inevitable and desirable) to interpret/understand/apply these norms in diverse ways in diverse contexts" (Ess, 2009, p. 191).

Taking an ethical pluralistic approach to pluralismin-the-small shows that it is possible to produce encyclopedic information that can be used in diverse cultures and represent different views on the same topic. However, it does require the abandonment of the current NPOV policy's notions of majority viewpoint, small minority viewpoints, and tiny minority viewpoints and instead requires the "wrestling and struggling with the fit of" (Smith, 1996, p. 108) the various ideas, values, notions, meanings, norms, 
opinions, and facts around a given topic. This means that Wikipedians ought to "observe diverse practices" (Ess, 2009, p. 192) as they encounter different cultures and that they "tolerate these differences... at least insofar as we can understand them to be different interpretations of a shared norm or value" ( $p$. 192). But Wikipedians should not "thereby tolerate any and all practices" (p. 192). In other words, Wikipedians ought to insist that there are indeed shared values, norms, and practices that are found across different cultures. Wikipedians have a moral duty to ensure that different views on a topic are observed and tolerated, and to write Wikipedia entries from the perspective of the shared values, norms, and practices such that all views are discussed from that perspective.

\section{Conclusions}

Wikipedia is not neutral. No encyclopedia, no knowledge, no explanation can be said to be neutral in the sense that is free of its authors' position in the world. The NPOV policy's reliance on trusted, reliable sources to distinguish between facts and opinions, and between majority and minority views does not free Wikipedians from their "makeup and position in the world" (Nagel, 1986, p. 5) when they construct entries for Wikipedia.

Every Wikipedian is situated in a language game, and is qua that position in the world able to know things about a given topic. However, that language game and that position shapes the Wikipedian's interpretation of the various views of that topic. Knowledge about and understandings of topics and the majority and minority views of the topic is shaped by and constructed from within particular language games. This does not mean that every Wikipedian has a subjective view of the topic, if by subjective we follow Nagel's terminology, but it does follow that no Wikipedian can claim an objective or neutral standpoint either. It does require Wikipedia and Wikipedians to recognize that they are active participants in the world and part of language games. As such, Wikipedians' claim to know something about a topic and the various viewpoints on that topic, takes place from within that language game, and the judgments of the status of various viewpoints on the topic is done from within that particular language game. Therefore, their judgment is per definition not neutral and without bias.
Wikipedians are curators of today's knowledge about the world. For many people Wikipedia is the first (and often last) resource consulted for information. This requires Wikipedians to act responsibly and, more importantly, it requires Wikipedia to formulate policies that do no harm and which take serious the important role that Wikipedia plays in society. While the current NPOV policy is written with the ideal of being accommodating to conflicting ideas and viewpoints by representing all "significant viewpoints" on a topic, it advances the notion that topics are valueneutral and true at the center and that a plural minority viewpoints exist only at the edges, outside comprehension within the majority viewpoint.

While the call for papers for this special issue noted the pronounced homogeneity of Wikipedians in terms of gender - but presumably also across other measurements - and suggested that a rise in the number of female Wikipedians might produce different kinds of entries and knowledge in Wikipedia, the argument in this paper is that the actual challenge lies within Wikipedia, within its NPOV policy. The NPOV policy is based on an understanding of knowledge, encyclopedias, and encyclopedic information where it matters not who produces and writes the entry. The first step for Wikipedia is to acknowledge that the locality of knowledge matters, that the languages games Wikipedians are part of matter, that knowledge should be likened to a rhizome with incommensurable points, that the challenges of inclusivity lie at the center and not the edges, and to explicitly take an ethical pluralistic position in its enterprise. My sense is that an authority who takes that position, who is humble about it, who allows people to wrestle and struggle with understanding the world, and who insists on a shared set of values, norms, and practices has the prospect of being recognized a cognitive authority across many cultures. This first step would require Wikipedia to re-write its NPOV policy.

\section{Notes}

1. Notwithstanding NPOV's suggestion to consult information resources of high quality, the notion of quality of information resources is in fact a topic that is debated and researched in the information studies literature - and there is no evidence to support the notion that the quality of information re- 
sources is as easily identified as the NPOV policy suggests (cf. e.g. Mai, 2013).

2. Leaving aside for a moment those who have improper influence on one's thoughts, such as advertisement and distributors of disinformation.

\section{References}

Eco, U (1984). Semiotics and the philosophy of language. London : Macmillan.

Ess, C (2009). Digital media ethics. Cambridge, UK: Polity Press.

Mai, J-E (2013). The quality and qualities of information. Journal of the American Society for Information Science and Technology, 64 (4), 675-688.

Nagel, T (1986). The view from nowhere. Oxford : Oxford University Press.
Smith, BC (1996). On the origin of objects. Cambridge, MA : MIT Press.

Wilson, P (1983). Second-hand knowledge: an inquiry into cognitive authority. Westport, CT : Greenwood Press.

Wikipedia (n.d.-a). Neutral point of view. Retrieved August 3, 2015, from https://en.wikipedia.org/wiki/ Wikipedia:Neutral_point_of_view

Wikipedia. (n.d.-b). Neutral point of view/FAQ. Retrieved August 3, 2015, from https://en.wikipedia. org/wiki/Wikipedia:Neutral_point_of_view/FAQ

Wittgenstein, L (1969). On certainty. Malden, MA : Blackwell Publishing. 